\title{
Optimal Control of Hybrid Electric Vehicles Based on Pontryagin's Minimum Principle
}

\author{
Namwook Kim, Sukwon Cha, Huei Peng
}

\begin{abstract}
A number of strategies for the power management of HEVs (Hybrid Electric Vehicles) are proposed in the literature. A key challenge is to achieve near-optimality while keeping the methodology simple. The Pontryagin's minimum principle (PMP) is suggested as a viable real-time strategy. In this paper, the global optimality of the principle under reasonable assumptions is described from a mathematical viewpoint. Instantaneous optimal control with an appropriate equivalent parameter for battery usage is shown to be possibly a global optimal solution under the assumption that the internal resistance and opencircuit voltage of a battery are independent of the state-of-charge (SOC). This paper also demonstrates that the optimality of the Equivalent Consumption Minimization Strategy (ECMS) results from the close relation of ECMS to the optimal-control-theoretic concept of PMP. In static simulation for a power-split hybrid vehicle, the fuel economy of the hybrid vehicle using the control algorithm proposed in this paper is found to be very close typically within $1 \%$ - to the fuel economy through global optimal control that is based on DP (Dynamic Programming).
\end{abstract}

Index Terms-road vehicle control, cost optimal control, fuel optimal control, dynamic programming, Pontryagin maximum principle.

\section{INTRODUCTION}

$\mathrm{T}$ he optimal control of HEVs (Hybrid Electric Vehicles) is an important topic not only because it is useful for powermanagement control but also indispensible for the optimal design of HEVs. Different vehicle systems can be compared to each other only when the controllers guarantee the optimality for each deployed system. Technically, we can obtain optimal control trajectories if the whole driving-cycle information is given prior, and if we have determinate performance indexes, such as fuel consumption, exhaust emission, or acceleration performance. Under those circumstances, the Dynamic Programming (DP) approach guarantees the global optimal results and had been investigated in several prior publications [1], [2], [3]. The results obtained through DP are unbeatable but, unfortunately, cannot be implemented directly. Instead, a post-processing step is required by using rule extraction, e.g., through Neural Networks, which approximates the results of the optimal control pattern. Even with this post-processing step, these strategies cannot cover all driving conditions. Hence, the real-time controller based on DP is effective only for the driving cycle that is used for rule extraction. To remedy this problem, stochastic dynamic programming and driving pattern detection with multiple driving cycles had been suggested as possible solutions [4], [5]. Another approach based on optimal control theory which, basically, realizes the minimization of the Hamiltonian has been applied to the optimal control problem for HEVs in [6], [7]. The Equivalent Consumption Minimization Strategy (ECMS), which really began from the heuristic concept that electric energy could be equivalent to fuel usage, was introduced [8]. Real-time applications of ECMS were suggested in [9], [10]. As a general case of the Euler-Lagrange equation, Pontryagin's minimum principle (PMP) was also introduced as an optimal control solution [11], [12], [13], wherein the Hamiltonian is considered as a mathematical function. In this paper, we show that the Hamiltonian can be calculated from numerical models and further, prove that the control concept based on PMP can be a global optimal solution under reasonable assumptions. The optimal control based on PMP is simple enough to be implemented in real-time applications because it is based on instantaneous optimization. Assuming that the cost function to be optimized involves only fuel consumption, the control concept minimizes the Hamiltonian, which is defined as:

$$
H=\dot{m}_{f c}\left(P_{b a t}\right)+p \cdot S \dot{O} C\left(S O C, P_{b a t}\right)
$$

where $\dot{m}_{f c}$ is the rate of fuel consumption, $p$ is an adjustment variable, which is called 'costate' in PMP, and $S \dot{O} C$ is a time derivative of $S O C$ (the state-of-charge).

\section{GENERAL APPROACHES FOR THE OPTIMAL CONTROL OF HEVS}

As stated above, assuming that minimum fuel consumption is the goal of optimal control, the problem of HEVs can be defined as (2), in which the engine speed, $S_{e}$, and the engine torque, $T_{e}$, can be used to determine the fuel consumption.

$$
\left\{\begin{array}{c}
\min \left\{J=\int_{t_{0}}^{t_{f}} L\left(S_{e}, T_{e}, t\right) d t\right\} \\
\text { subject to }: \\
\operatorname{SOC}\left(t_{0}\right)=\operatorname{SOC}\left(t_{f}\right) \\
S \dot{S} C=f\left(S O C, S_{e}, T_{e}\right) \\
S O C_{\min } \leq S O C \leq S O C_{\max } \\
S_{\min } \leq S_{e} \leq S_{\max } \\
T_{\min } \leq T_{e} \leq T_{\max }
\end{array}\right.
$$


where $L\left(S_{e}, T_{e}\right)$ is the rate of fuel consumption of the engine. $S O C$ is determined by a battery model, which will be described in (8). Further, $T_{e}$ and $S_{e}$ are restricted by operating constraints such as the maximum possible engine speed or the maximum possible engine torque given the impact of constraints on components, such as the maximum motor speed maximum torque, or maximum battery power. This optimal control problem can be solved from optimal control techniques, which are described in the next section.

\section{A. Optimal Control Theory}

To solve a deterministic optimal control problem, which is defined as (2), there are two representative approaches. One is the Hamilton-Jacobi-Bellman (HJB) approach, which is based on Bellman's principle of optimality, and the other is trajectory optimization, which originates from the Calculus of Variation.

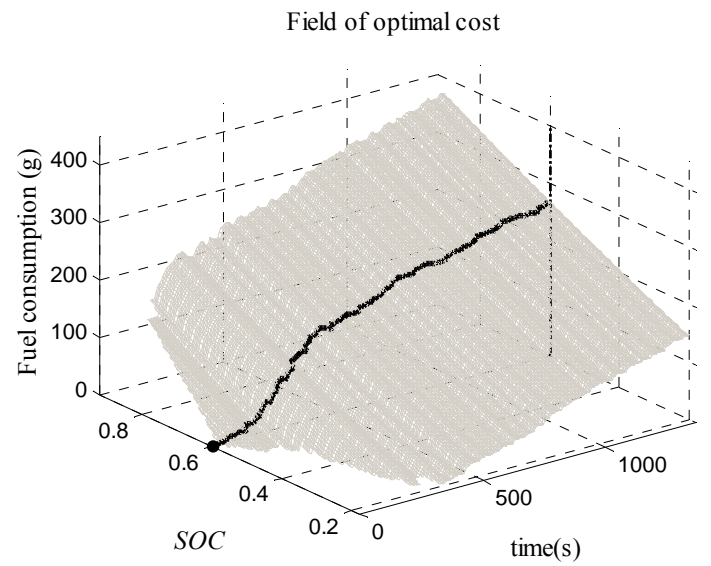

Fig. 1. A field of the optimal cost. The field is a family of optimal fuel consumptions. The starting point is $S O C=0.6$ and $t=0$. Contrary to this figure, in general, a field of cost-to-go is widely used in optimal control problems because it is more useful for dealing with state equations.

As a kind of numerical method for the HJB equation, Dynamic Programming (DP) solves a field of optimal control that is based on the principle of optimality; the field is a family of optimal fuel consumptions, as shown in Fig. 1. On the other hand, Pontryagin's minimum principle (PMP), which is a general case of the Euler-Lagrange equation in the Calculus of Variation, considers the optimality of a single trajectory. (See Fig. 2.)

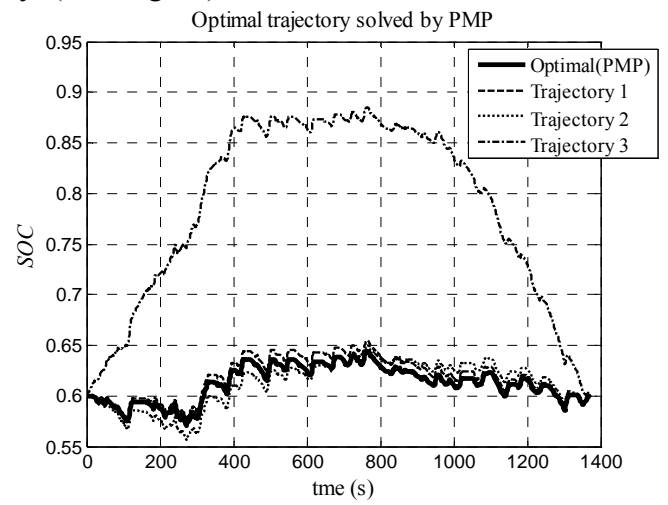

Fig. 2. The trajectory derived from PMP. The trajectory is superior to only neighboring trajectories (i.e., it is only locally optimal), which means the trajectory from PMP could be inferior to Trajectory 3 , which is not adjacent to the PMP-derived trajectory.

In general, the DP approach guarantees the global optimal solution by obtaining all possible optimal trajectories from the field of optimal control. On the other hand, PMP, as one method of trajectory optimization, yields us necessary - but not sufficient - conditions that the absolute (i.e., global optimal) trajectory must satisfy. Hence, there could be a superior solution that is distant from the local optimal trajectory that is obtained from PMP. (See Fig. 2.)

\section{B. DP vs. PMP}

As has been stated above, the trajectory derived from PMP might not be a global optimal solution. Therefore, the control based on PMP can be considered as inferior to the (globally optimal) control based on DP. On the other hand, DP requires more computing time than PMP because DP solves all possible optimal controls to fill the optimal field [11]. Since DP is a numerical representation of the HJB equation, DP needs a similar computation load as the HJB equation, which solves a partial differential equation (PDE), whereas PMP solves just nonlinear second-order differential equations. The drawback of DP with regard to the computational load becomes compounded due to the 'curse of dimensionality,' i.e., when the state variables increase in number, the computational load of the PDE exponentially increases in accordance with the increase in the dimension of the optimal field. However, in PMP, the number of nonlinear second-order differential equations linearly increases with the dimension. In conclusion, we can say that the control based on PMP can reduce the computational time for getting an optimal trajectory but it could be a local optimal solution, not a global solution in general problems.

\section{Sufficient Conditions for Global Optimality of PMP}

In specific cases, the optimal control based on PMP can be a global optimal control. For example, it is well-known that the optimal control based on the Euler-Lagrange equation can be a global optimal control in a linear system [15]. In general, the following three approaches are effective to establish that the necessary conditions from PMP become sufficient for the global optimal control: 1) the optimal trajectory obtained from PMP is a unique trajectory that satisfies the necessary and boundary conditions; 2) some geometrical properties of the optimal field provide the possibility of optimality verification; and 3) as a general statement of the second approach, the absolute optimality is, mathematically, proved by clear propositions [21]. If one of these three approaches is applicable to the optimal control problem of HEVs, we can replace DP with PMP, which can save on time to yield optimal results and also guarantee global optimality. 


\section{APPLICATION OF PONTRYAGIN’S MINIMUM PRINCIPLE}

In the optimal control problem of HEVs, Pontryagin's minimum principle (PMP) produces a boundary value problem within second-order nonlinear differential equations. In this section, we introduce a static model of a target vehicle and describe the techniques for obtaining the Hamiltonian from the model and deciding on the optimal control from the Hamiltonian, which is a local process of getting the optimal trajectory through PMP.

\section{A. Vehicle Model}

In this paper, we assume that the hybrid vehicle uses a powersplit hybrid, viz., the Toyota Hybrid System (THS), which integrates two motors/generators and an engine through a planetary gear set (see Fig. 3). In the static model of the power-split system, we have two independent control variables, the torque of the engine and the speed of the engine, when the requested output speed and torque are given, which means all other variables can be fixed by these two control variables.

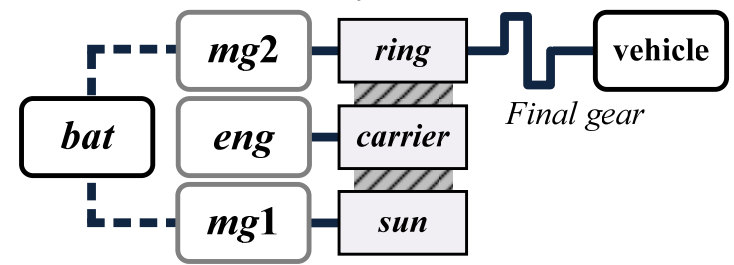

Fig. 3. A schematic diagram of a power-split hybrid system considering the final differential gear ratio.

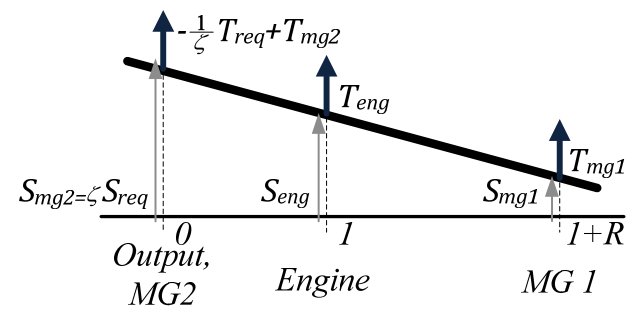

Fig. 4. The lever diagram for the lever analogy of the THS system by which the force and momentum equilibria are simply obtained.

The torque and speed relations among the power resources are well-known under static conditions [2]. We can obtain the operating torques and speeds of the MG1 and MG2, which are functions of the engine torque and the engine speed, when the requested output torque and speed are already calculated from the driving cycle.

$$
\begin{gathered}
{\left[\begin{array}{l}
T_{m g 1} \\
T_{m g 2}
\end{array}\right]=\frac{1}{-(1+R)}\left[\begin{array}{cc}
0 & 1 \\
1+R & R
\end{array}\right] \cdot\left[\begin{array}{c}
-1 / \zeta \cdot T_{r e q} \\
T_{e n g}
\end{array}\right]} \\
{\left[\begin{array}{l}
S_{m g 1} \\
S_{m g 2}
\end{array}\right]=\left[\begin{array}{cc}
-R & 1+R \\
1 & 0
\end{array}\right] \cdot\left[\begin{array}{c}
\zeta \cdot S_{r e q} \\
S_{e n g}
\end{array}\right]}
\end{gathered}
$$

where $T_{\text {eng }}, T_{m g 1}, T_{m g 2}$, and $T_{\text {req }}$ are the torques of the engine, MG1, and MG2, and the requested torque of output, respectively. Further, $R$ and $\zeta$ are the gear ratio of the planetary gear set and the final gear ratio, respectively. $S_{e n g}, S_{m g 1}, S_{m g 2}$, and $S_{\text {req }}$ are the speeds of the engine, MG1, and MG2, and the requested output speed, respectively. The required power of the battery can then be calculated as:

$$
P_{b a t}=\eta_{c 1}{ }^{k} \cdot T_{m g 1} \cdot S_{m g 1}+\eta_{c 2}{ }^{k} \cdot T_{m g 2} \cdot S_{m g 2}
$$

where the efficiencies of MG1 and MG2, $\eta_{c 1}$ and $\eta_{c 2}$, are obtained based on motor efficiency maps of each MG, which include the motor and inverter losses, and

$$
k=\left\{\begin{array}{cc}
1, & \text { recuperating } \\
-1, & \text { motoring }
\end{array}\right.
$$

We can also use a fuel consumption rate map to obtain $\dot{m}_{f c}$.

$$
\dot{m}_{f c}=L\left(T_{\text {eng }}, S_{\text {eng }}\right)
$$

Finally, the derivative of $S O C, S O C$ can be calculated from the battery power and the current SOC. Considering that the equivalent open-circuit voltage and internal resistance are functions of $S O C, S O C$ is a function of $S O C$ and $P_{b a t}$, which can be expressed as [2].

$$
S \dot{O} C=\frac{1}{Q_{b a t}} \cdot \frac{V_{b a t}-\sqrt{V_{b a t}^{2}-4 R_{b a t} P_{b a t}}}{2 R_{b a t}}
$$

In conclusion, $\dot{m}_{f c}$ in (7) and $S \dot{O} C$ in (8) depend on $T_{e n g}, S_{\text {eng }}$, and $S O C$, when the requested output condition is given.

\section{B. Confined Optimal Operating Line (C-OOL)}

Prior to solving the time horizon optimal problem of HEV, we introduce an inner-loop optimal process to get ConfinedOptimal Operating Line (C-OOL), which is the family of the best engine operating points confined to specific output torque and speed. This optimal process makes the Hamiltonian as a function of only one control variable, $P_{b a t}$. To get the Hamiltonian, first, we calculate $\dot{m}_{f c}$ and $P_{b a t}$ which are functions of the control variables, $T_{\text {eng }}$ and $S_{\text {eng }}$ described in (7) and (9) from the static model (3)-(5) in every second.

$$
P_{b a t}=h\left(T_{\text {eng, }} S_{\text {eng }}\right)
$$

The optimal problem to minimize the fuel consumption subject to a specific battery power, $P_{b a t}$, is defined as:

$$
\begin{array}{ll}
\min & {\left[\dot{m}_{f c}=L\left(T_{\text {eng }}, S_{\text {eng }}\right)\right]} \\
\text { s. t. } & h\left(T_{\text {eng, }} S_{\text {eng }}\right)-P_{\text {bat }}=0 \\
& \text { Optimal points for C-OOL }
\end{array}
$$

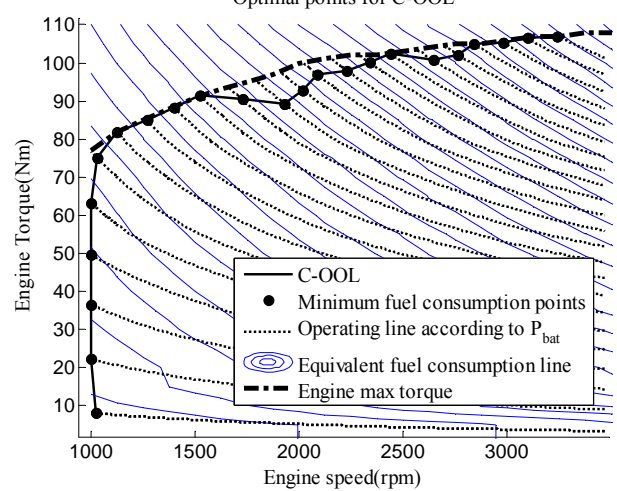

ig. 5. An example of C-OOL when $T_{\text {req }}=100 \mathrm{Nm}$ and $S_{\text {req }}=100 \mathrm{rad} / \mathrm{s}$. Dottedlines are feasible operating lines for specific values of $P_{b a t}$. The resolution of $P_{b a t}$ in the figure is $1.5 \mathrm{~kW}$ whereas it is $0.05 \mathrm{~kW}$ in our simulation. 
The optimized control variables, $T_{e n g}^{*}$ and $S_{e n g}^{*}$, in the problem can be decided by choosing a minimum point of fuel consumption on each feasible engine operating line as per $P_{b a t}$ (see the dotted-line in Fig. 5). Finally, the instantaneous optimal fuel consumption rate is a function of the optimized control variables, $T_{e n g}^{*}$ and $S_{\text {eng }}^{*}$, subject to the specified $P_{\text {bat }}$.

$$
\begin{aligned}
& \dot{m}_{f c}=L\left(T_{\text {eng }}^{*}, S_{\text {eng }}^{*}\right) \\
& P_{b a t}=h\left(T_{\text {eng }}^{*}, S_{\text {eng }}^{*}\right)
\end{aligned}
$$

In light of the time-horizon optimal control, this optimal process reduces the dimension of the control variable from $T_{\text {eng }}$ and $S_{\text {eng }}$ to only $P_{b a t}$, whereby we do not consider the inferior engine operating points when solving the problem in the time-horizon plane. Now, from (11) and (12), we obtain the fuel consumption, $\dot{m}_{f c}$, which is a function of $P_{b a t}$, as shown in Fig. 6.

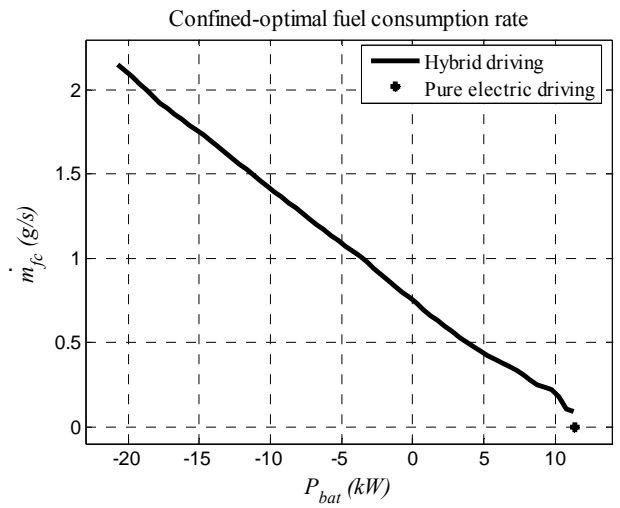

Fig. 6. Instantaneous optimal fuel consumption rate line in the domain set, $\left\{h\left(T_{r e q}, S_{\text {req }}\right), L\left(T_{r e q}, S_{r e q}\right)\left[T_{r e q}, S_{r e q}\right] \in\left[T_{r e q}^{*}, S_{r e q}^{*}\right]\right\}$, when $T_{r e q}=100 \mathrm{Nm}$ and $S_{\text {req }}=100 \mathrm{rad} / \mathrm{s}$.

Now, the fuel consumption rate, $\dot{m}_{f c}$, can be determined by $P_{b a t}$, which could be decided by a supervisory algorithm. Then, the engine operating point and all other operating points of power resources are calculated based on C-OOL. The optimal fuel consumption rate line in Fig. 6 can be interpreted as a kind of Pareto frontier for all the engine operating points [22]. The physical interpretation of the line in Fig. 6 is clear: given that the engine always operates at the best point, less fuel consumption is needed when more battery power is used, and vice versa. In general, the requested output torque and speed vary over time; hence, we can assert that in the time-horizon plane, the fuel consumption rate, $\dot{m}_{f c}$, is a function of just $P_{b a t}$ and $t$, as in (13).

$$
\dot{m}_{f c}=g\left(P_{b a t}, t\right)
$$

Additionally, the pure electric driving point shown in Fig. 6 is the operating point at which the battery supplies all the energy needed to drive the vehicle while the engine does not operate or, if appropriate, operates at an optimal speed with no fuel consumption but with engine drag.

\section{Necessary Conditions from PMP}

From the assistance of the inner-loop optimal process, only $P_{b a t}$ is the control variable that decides all the operating points in the time-horizon plane of the optimal control problem. The control variable, $P_{b a t}$, decides the fuel consumption rate and the engine operating point in the C-OOL. All the other system variables, such as the motor speed, torque, and transmission status, are fixed from the engine operating point. To obtain the optimal $P_{b a t}$, the performance index can be defined as:

$$
\min \left[J=\int_{t_{0}}^{t_{f}} g\left(P_{b a t}(t), t\right) d t\right]
$$

In (14), $g$ is the best fuel consumption rate function in (13), which is a function of $P_{b a t}$ and $t$. From PMP, the Hamiltonian is defined as (15), where $p$ is the costate.

$$
H=g\left(P_{b a t}\right)+p \cdot f\left(S O C, P_{b a t}\right)
$$

The state equation and the costate equation can be expressed as (16) and (17), respectively.

$$
\begin{gathered}
S \dot{O} C=\partial H / \partial p=f\left(S O C, P_{b a t}\right) \\
\dot{p}=-\partial H / \partial(S O C)=-p \cdot \partial f / \partial(S O C)
\end{gathered}
$$

For optimality, another condition in (18) should be considered to determine the optimal control variable, $P_{b a t}$, at every time step.

$$
H\left(P_{b a t}^{*}, S O C^{*}, p^{*}, t\right) \leq H\left(P_{b a t}, S O C^{*}, p^{*}, t\right)
$$

Finally, when both the final time and the final state are fixed, as in the optimal control problem of HEVs, the boundary condition of the final state is added for the sake of optimality.

$$
\operatorname{SOC}\left(t_{0}\right)=\operatorname{SOC}\left(t_{f}\right)
$$

These conditions, (16) (19), are necessary and boundary conditions that the optimal trajectory must satisfy [19].

\section{Optimal Control using the Hamiltonian}

The Hamiltonian is obtained from Eq. (13), Eq. (8), and the costates. The optimal control is determined by the necessary condition in (18). Fig. 7 shows three examples of the Hamiltonian and the associated optimal controls. We can choose the optimal control, $P_{b a t}$, which minimizes the Hamiltonian if the costate is given beforehand, though the optimal costate is obtained over the entire time-horizon time to satisfy the boundary condition in (19).

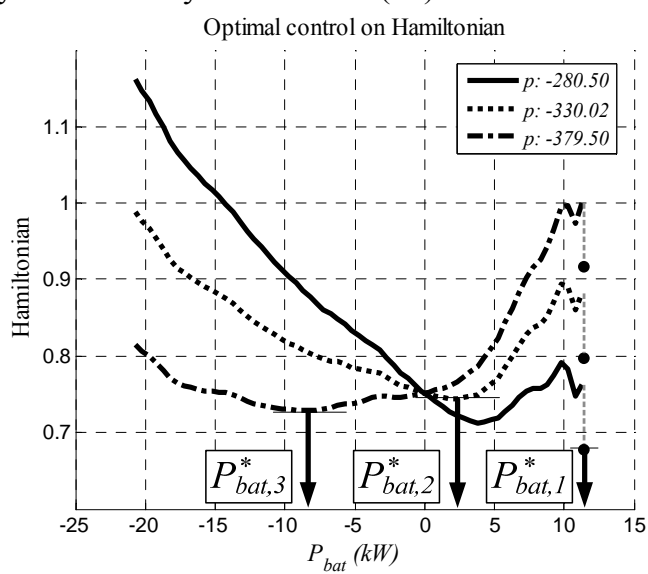

Fig. 7. Three examples of the Hamiltonian and the associated optimal controls for varying costates when $T_{\text {req }}=100 \mathrm{Nm}, T_{\text {req }}=100 \mathrm{Nm}, S_{\text {req }}=100 \mathrm{rad} / \mathrm{s}$, and $\mathrm{SOC}$ 
is 0.6 .

If we have an appropriate costate, this instantaneous optimal control could be an optimal solution in the timehorizon control problem and the Hamiltonian function would not need to be an explicit function. In Fig. 7, the higher costate makes the controller choose the higher $P_{b a t}^{*}$ as an optimal control, which lowers SOC. Additionally, the Hamiltonian in Fig. 7 is an almost-convex function and the constraint on the state is presented by a linear summation of SOC. In that case, mathematically, the optimization problem possibly possesses an appropriate costate, which implies that we can find the appropriate costate to satisfy the boundary condition; this is a situation of strong duality in optimization [23]. As shown in Fig. 7, the control concept is based on instantaneous minimization but the control can be optimal only when an appropriate costate is given.

\section{CHARACTERISTICS OF THE HEV PROBLEM}

In view of optimal control, the HEV problem has special characteristics. The fuel consumption rate in Fig. 6 is not a function of the state variable, $S O C$, and $S \dot{O} C$ in (16) is not only independent of time but also highly depends on $P_{b a t}$ rather than SOC. These characteristics influence the properties of the solution from PMP. In this section, we describe the specialties of the optimal control problem of HEVs with regard to the above characteristics. We also introduce several techniques to apply PMP in general problems of HEVs.

\section{A. Constant Costate}

The costate in (15) originates from Lagrange multipliers for the incorporation of dynamic constraints, which, occasionally, possess physical meanings. In the HEV problem, the costate can be interpreted as a 'weight' coefficient of the time derivative of $S O C$, by which the second term in (15) can be interpreted as an equivalent fuel consumption. On the other hand, the costate in our problem can be considered as a constant under some assumptions about the battery. In general, the $S O C$ range of the battery usage is limited between 0.2 and 0.9 but in charge-sustaining problems, the battery mainly operates in a narrower range, e.g., from 0.5 to 0.7 ; hence, the voltage and the resistance may not vary so much in the range.

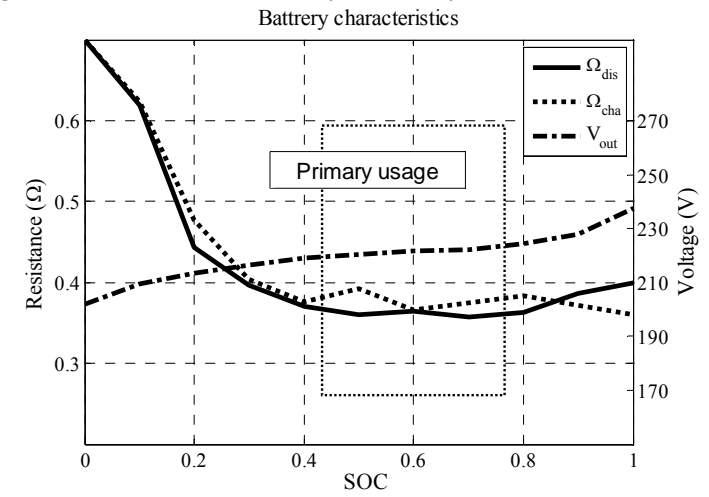

Fig. 8. Plot of the battery's open-circuit voltage and resistance (used in Prius04).
In that case, the costate stays near the initial value because, mathematically, the absolute value of $\partial f / \partial(S O C)$ in (17) is very small when compared to the absolute value of the costate for the entire driving cycle. If the resistance and the voltage are constants or depend on only $P_{b a t}$, we can assume that $S \dot{O} C$ depends on only $P_{b a t}$ and not on SOC. Then, the costate expression, i.e., (17), vanishes in our optimal control problem, which is reasonable in the primary range of usage of $S O C$.

$$
S \dot{O} C=f\left(S O C, P_{b a t}\right) \simeq f\left(P_{b a t}\right)
$$

Now, the costate can be considered as a constant.

$$
\dot{p}=-p \cdot \partial f / \partial(S O C)=0, \quad \rightarrow p=\text { constant }
$$

Owing to the constant costate, we do not only reduce the complexity of the optimal control based on PMP but also discover an interesting feature of the optimal control problem of HEVs.

\section{B. Condition of Global Optimality}

The idea of assuming the costate as constant is suggested in several prior studies to simplify the computations in simulation; the assumption has been described as an intuitively reasonable assumption [6], [10], [14]. However, the essential point of the assumption is that the optimal control based on PMP can become a global optimal control under the assumption. To prove this proposition, we have to return to the first approach of section II.C. As stated in that section, if an optimal trajectory that satisfies the necessary conditions of PMP and the boundary conditions is unique, the optimal trajectory should be considered as a global optimal trajectory. In our problem, we can consider two distinct trajectories that have different costates but satisfy all the necessary conditions and the boundary condition in (16) (19) shown in Fig. 9. Under the assumptions of the battery, the Hamiltonian can be presented as:

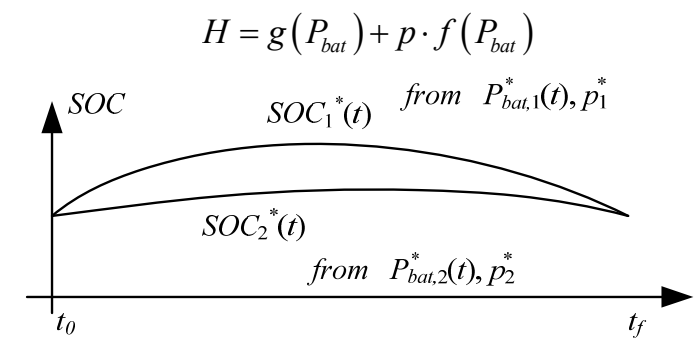

Fig. 9. The two optimal SOC trajectories that satisfy the necessary conditions and the boundary condition.

From the necessary condition in Eq. (18), the optimal control variable for $S O C_{1}^{*}, P_{b a t, 1}^{*}$, satisfies the condition in Eq. (23).

$$
H\left(P_{b a t, 1}^{*}, p_{1}^{*}, t\right) \leq H\left(P_{b a t}, p_{1}^{*}, t\right)
$$

This condition should be satisfied for all admissible values of $P_{b a t}$ shown in Fig. 7, including $P_{b a t, 2}^{*}$, i.e., either

$$
H\left(P_{b a t, 1}^{*}, p_{1}^{*}, t\right) \leq H\left(P_{b a t, 2}^{*}, p_{1}^{*}, t\right),
$$




$$
g\left(P_{b a t, 1}^{*}, t\right)+p_{1}^{*} \cdot f\left(P_{b a t, 1}^{*}\right) \leq g\left(P_{b a t, 2}^{*}, t\right)+p_{1}^{*} \cdot f\left(P_{b a t, 2}^{*}\right),
$$

We can apply the same argument for $S O C_{2}^{*}$, which can be expressed as:

$$
g\left(P_{b a t, 2}^{*}, t\right)+p_{2}^{*} \cdot f\left(P_{b a t, 2}^{*}\right) \leq g\left(P_{b a t, 1}^{*}, t\right)+p_{2}^{*} \cdot f\left(P_{b a t, 1}^{*}\right) .
$$

Another inequality is obtained by summing the above two inequalities; this can be expressed as:

$$
\left(p_{1}^{*}-p_{2}^{*}\right) \cdot\left\{f\left(P_{b a t, 1}^{*}\right)-f\left(P_{b a t, 2}^{*}\right)\right\} \leq 0 .
$$

Now, we can replace the state equation, $f\left(P_{b a t}\right)$, with $S \dot{O} C$.

$$
\left(p_{1}^{*}-p_{2}^{*}\right) \cdot\left(S \dot{O} C_{1}^{*}-S \dot{O} C_{2}^{*}\right) \leq 0 \text {. }
$$

The condition in Eq. (28) indicates that the two existing optimal trajectories, which have the same initial and final $S O C$ values, are impossible because the sign of $\left(S \dot{O} C_{1}^{*}-S \dot{O} C_{2}^{*}\right)$ does not change for all $t$ under different constant costates. Finally, the supposition that there are two different optimal trajectories that satisfy both the necessary and the boundary conditions is refuted. Additionally, from Eq. (28), we can derive the proposition that the $S O C$ trajectory that has a higher costate always either increases at a slower rate or decreases at a faster rate than the trajectory with a lower costate under the assumptions of the battery, which makes sense in light of the observations on the optimal control of the Hamiltonian in section III.D.

\section{State Variable Constraints}

The constraints on the control variable, $P_{b a t}$, can be applied to the optimal problem when calculating the Hamiltonian from the static model. The constraints on the state variable, such as maximum or minimum limits on $S O C$, however, are not introduced above. If needed, we can apply a state variable inequality constraint by adding a new imaginary state variable, $S O C_{C}$, and augmenting the Hamiltonian with the adjusted terms. To apply the constraint, we have to define the state equation of $S O C_{C}$ as in [19].

$$
\begin{aligned}
S O C_{C}= & \left(S O C-S O C_{\min }\right)^{2} \times \mathrm{U}\left(S O C_{\text {min }}-S O C\right) \\
& +\left(S O C_{\text {max }}-S O C\right)^{2} \times \mathrm{U}\left(S O C-S O C_{\text {max }}\right)
\end{aligned}
$$

In (29), $\mathrm{U}$ is a unit Heaviside step function and $S O C_{\min }$ and $S O C_{\max }$ are minimum and maximum limits on $S O C$, i.e., 0.2 and 0.9. If we set both $S O C_{C}\left(t_{0}\right)$ and $S O C_{C}\left(t_{f}\right)$ to zero, the imaginary state variable, $S O C_{C}$, becomes zero for the entire driving cycle because $S \dot{O} C_{C}$ can never be nonzero when the state does not violate the constraint on $S O C$ for the entire driving cycle. The Hamiltonian, including the augmented term about $S O C_{C}$, is defined as:

$$
H=\dot{m}_{f c}+p \cdot S \dot{O O C}+p_{C} \cdot S \dot{O O} C_{C}
$$

From PMP, the costate equation corresponding to the new state can be expressed as:

$$
\dot{p}_{C}=-\partial H / \partial\left(S O C_{C}\right)=0
$$

The state equation and the costate equation, (29) and (31), should be added to the original necessary conditions of PMP.
This additional state is trivial in the optimal control problem for a charge-sustaining HEV because there is little likelihood that its optimal SOC trajectory violates the SOC limitation. This consideration, however, is effective in the optimal control problem for plug-in hybrid vehicles.

\section{Cost Function}

In the PMP algorithm, the Hamiltonian can be modified if we have to consider new components of cost, such as emissions or the estimation of drivability, or new states, such as the temperature. The performance index of the problem with these new costs and states can be defined as:

$$
J=h\left(\mathbf{x}\left(t_{f}\right), t_{f}\right)+\int_{t_{0}}^{t_{f}}\left(\dot{m}_{f c}+g_{\text {new }}\right) d t
$$

In (32), $g_{\text {new }}$ is a new cost function, $\mathbf{x}$ is a new state, and $h$ is a cost function for the final states. The modified Hamiltonian can be expressed as:

$$
H=\dot{m}_{f c}+g_{\text {new }}+p \cdot S \dot{O O C}+\mathbf{p}_{\text {new }}^{\mathrm{T}}(t) \cdot \dot{\mathbf{x}}_{\text {new }}(t)
$$

In (33), $\mathbf{p}_{\text {new }}$ is a new costate vector. The necessary conditions of PMP, (16) (18), can be also applied for the new Hamiltonian. In general, if the final state of the new states, $\mathbf{x}\left(t_{f}\right)$, is not fixed, the new boundary condition, (34), should be added instead of the state boundary condition for the fixed boundary [19].

$$
\left[\partial h / \partial \mathbf{x}_{\text {new }}-\mathbf{p}_{\text {new }}\right]_{t_{f}}=0
$$

If the following two conditions are satisfied, this addition of new costs does not affect the global optimality of PMP, which means that the uniqueness that we established in section IV.B is still applicable. 1) The new cost is not a function of the state variables, $S O C$ or $\mathbf{x}_{\text {new }}$; therefore, the cost can be directly calculated from the control variable, $P_{b a t}$, i.e., the new cost is determined by engine operating points, as is the case with the fuel consumption. 2) There is no state except SOC, or, if there is such a state, the state equations are not functions of the state variables at all. In this case, we can apply the same argument to the new Hamiltonian, for which we can consider two distinct trajectory vectors, $\mathbf{x}_{1}$ and $\mathbf{x}_{2}$, which satisfy the necessary condition in Eq. (18). Now, we have a similar result as with Eqs. (23) (28). The result is expressed as:

$$
\left(\mathbf{p}_{1}^{*}-\mathbf{p}_{2}^{*}\right) \cdot\left(\dot{\mathbf{x}}_{1}^{*}-\dot{\mathbf{x}}_{2}^{*}\right) \leq 0
$$

In (35), $\mathbf{p}^{*}$ is a constant costate vector that includes $p$ and $\mathbf{p}_{\text {new }}$ and $\mathbf{x}^{*}$ is a state vector that includes $S O C$ and the new state, $\mathbf{x}_{\text {new }}$. When $\mathbf{x}_{1}^{*}\left(t_{0}\right)=\mathbf{x}_{2}^{*}\left(t_{0}\right)$, there is no possibility that $\mathbf{x}_{1}^{*}\left(t_{f}\right)=\mathbf{x}_{2}^{*}$ $\left(t_{f}\right)$ except that

$$
\left(\mathbf{p}_{1}^{*}-\mathbf{p}_{2}^{*}\right) \cdot\left(\dot{\mathbf{x}}_{1}^{*}-\dot{\mathbf{x}}_{2}^{*}\right)=0, \quad \forall t
$$

The inequality in (35) is an equality, i.e., $\left(\mathbf{p}_{1}^{*}-\mathbf{p}_{1}^{*}\right)$ is an orthogonal vector of $\left(\dot{\mathbf{x}}_{1}^{*}-\dot{\mathbf{x}}_{2}^{*}\right)$, only when the minimum Hamiltonian for $\mathbf{x}_{1}$ is coincident with the minimum Hamiltonian for $\mathbf{x}_{2}$ for all time-points, which is an unusual situation. Therefore, we can conclude that $\mathbf{x}_{1}^{*}(t)=\mathbf{x}_{2}^{*}(t)$ for all $t$ is the only solution if the final trajectory vector, $\mathbf{x}_{1}^{*}\left(t_{f}\right)$, equals $\mathbf{x}_{2}^{*}\left(t_{f}\right)$ when $\mathbf{x}_{1}^{*}\left(t_{0}\right)=\mathbf{x}_{2}^{*}\left(t_{0}\right)$. This proposition states that the 
optimal trajectory $\mathbf{x}^{*}$ is still unique even though new cost functions may be added. In general, if there are additional costs or states, such as emission costs or the temperature, either the cost can be a function of the new states or the states might be coupled to each other. Then, the optimal control based on PMP cannot guarantee global optimal control though it remains the case the control based on PMP is locally optimal.

\section{E. ECMS and PMP}

The equivalent consumption minimization strategy (ECMS) was introduced as an optimal control idea. Several control strategies based on ECMS, such as Adaptive-ECMS and Telemetric-ECMS, were suggested as real-time optimal control concepts in [8], [9], [10].

$$
\min \left[E F C=\left\{\begin{array}{ll}
\dot{m}_{f c}+s_{c h a} P_{b a t}, & P_{b a t}>0 \\
\dot{m}_{f c}+s_{d i s} P_{b a t}, & P_{b a t}<0
\end{array}\right]\right.
$$

The performance index of ECMS in (37) is generally called the equivalent fuel consumption (EFC) though the forms of application of the idea vary slightly. ECMS was really developed from a heuristic concept that current battery usage would be compensated for in the future; so, there are two different coefficients, $s_{c h a}$ and $s_{d i s}$, for the charging and discharging statuses, which influence the electric-energy balance. From the similarity between the Hamiltonian and EFC, ECMS was described as being fundamentally linked to the Euler-Lagrange equation [14]. It is natural to view ECMS from the concept of PMP because PMP states that the Hamiltonian does not have to be an analytic or explicit function. From the comparison between the Hamiltonian and EFC,

$$
p \cdot S \dot{O} C \approx \begin{cases}s_{c h a} P_{b a t}, & P_{b a t}>0 \\ s_{d i s} P_{b a t}, & P_{b a t}<0\end{cases}
$$

When the equivalent factors in ECMS are optimized for the HEV problem, these factors, $s_{c h a}$ and $s_{d i s}$, can be linked to the optimal costate of PMP, as in Eq. (38).

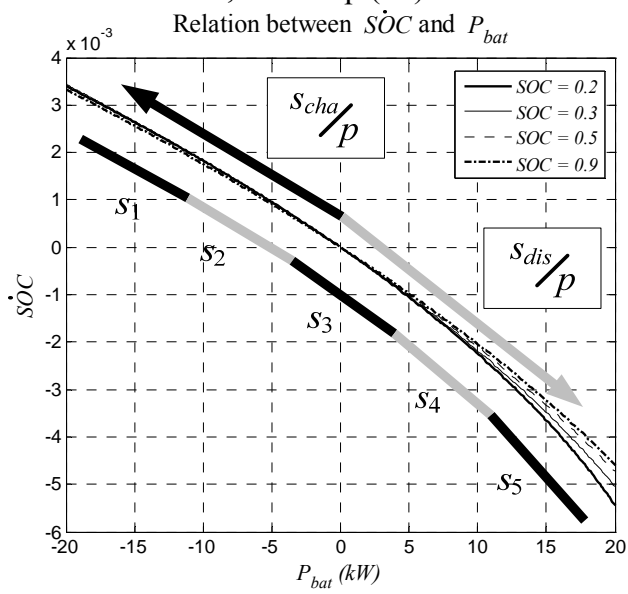

Fig. 10. The correlation between $S \dot{O} C$ and $P_{b a t}$ from the battery model. The equivalent factors in ECMS come from the mean slopes of the charging and discharging statuses

Fig. 10 shows the correlation between $P_{b a t}$ and $S \dot{O} C$ as per the $S O C$ from the battery model in (8). When the two equivalent coefficients of ECMS, $s_{c h a}$ and $s_{d i s}$, are optimized for the entire driving cycle to satisfy the energy balance, the boundary condition, $\operatorname{SOC}\left(t_{0}\right)=\operatorname{SOC}\left(t_{f}\right)$, is satisfied. These two coefficients are linked to the charging and discharging slopes in Fig. 10. By the use of two parameters rather than one, the EFC can be brought closer to the Hamiltonian. If more distinct and equivalent parameters are used, such as $s_{1}, s_{2}, s_{3}, s_{4}$, and $s_{5}$, as in Fig. 10, the closer is the fuel consumption of ECMS to the optimal value. On the other hand, in the optimal control based on ECMS, if the correlation between $P_{b a t}$ and $S \dot{O} C$ is too complicated to be represented by these two slopes, ECMS might not show good results, as in [9], [24]. On the other hand, the optimal control based on PMP is not affected by the complexity of the correlation between $P_{b a t}$ and $S \dot{O} C$. In conclusion, ECMS is closely connected to optimal control theory because EFC can be considered as a Hamiltonian-like function but the optimal control based on exact Hamiltonians could be better in the sense that it guarantees optimality, regardless of the complexity of the correlation between $P_{b a t}$ and $S \dot{O} C$.

\section{OPTIMAL SIMULATION}

For simulation, all vehicle data come from the model of Prius 04 in the Powertrain System Analysis Toolkit (PSAT). As a numerical method of PMP, the variation of extremals with multiple shooting methods, as in [20], is used to get the optimal trajectory that satisfies the necessary conditions. Based on the simulation results, we can consider that the assumptions of the battery are reasonable in charge-sustaining problems.

Table 1. Vehicle parameters applied in the simulation. All parameters are based on PSAT, except for the total vehicle mass and the transmission gear efficiency.

\begin{tabular}{l|l}
\hline Vehicle total mass & $1405 \mathrm{~kg}(1$ persons $)$ \\
\hline Engine & $\mathrm{Si} 1497 \_57 \_$US_04Prius \\
\hline Motor1 & pm_25_50_prius \\
\hline Motor2 & pm_15_30_prius \\
\hline Battery & Nimh_6_168_panasonic_MY04_Prius \\
\hline Planetary gear ratio & $2.6(78 / 30)$ \\
\hline Final gear ratio & 4.113 \\
\hline Transmission gear efficiency & $90 \%$ \\
\hline Rolling resistance coefficient & $0.007+(0.00012 \cdot$ vehicl velocity $)$ \\
\hline Frontal area & $1.746 \mathrm{~m}^{2}$ \\
\hline Drag coefficient & 0.29 \\
\hline Wheel radius & $0.305 \mathrm{~m}$ \\
\hline Air density & $1.23 \mathrm{~kg} / \mathrm{m}^{3}$ \\
\hline SOC resolution & 0.00001 \\
\hline Engine torque resolution & $1 \mathrm{Nm}$ \\
\hline Engine speed resolution & $1 \mathrm{rad} / \mathrm{s}$ \\
\hline
\end{tabular}

\section{A. Results from PMP and DP}

There are three optimal SOC trajectories in Fig. 11, which are the optimal SOC trajectory from DP, the optimal trajectory from PMP, and the optimal trajectory from PMP when a constant costate is used under the Federal Urban Driving Schedule (FUDS). First of all, the simulation results in Table 2 show that the fuel consumptions under PMP are very close to 
the results of DP.

Table 2. Results on fuel economy through DP and PMP.

\begin{tabular}{c|c|c|c}
\hline \multirow{2}{*}{ cycle name } & \multirow{2}{*}{ DP $(\mathrm{km} / \mathrm{l})$} & \multicolumn{2}{|c}{ PMP $(\mathrm{km} / \mathrm{l})$} \\
\cline { 3 - 4 } & & Exact solution & Constant $p$ \\
\hline FUDS & 34.6597 & 34.6364 & 34.6361 \\
\hline
\end{tabular}

The total fuel consumption through PMP is $0.07 \%$ less than through DP. If the truncation error is considered, it is hard to conclude that there is a significant difference. The optimal trajectories of SOC from DP, PMP, and PMP with a constant costate in Fig. 11, show that the trends in $S O C$ usage from these control algorithms are nearly coincident.

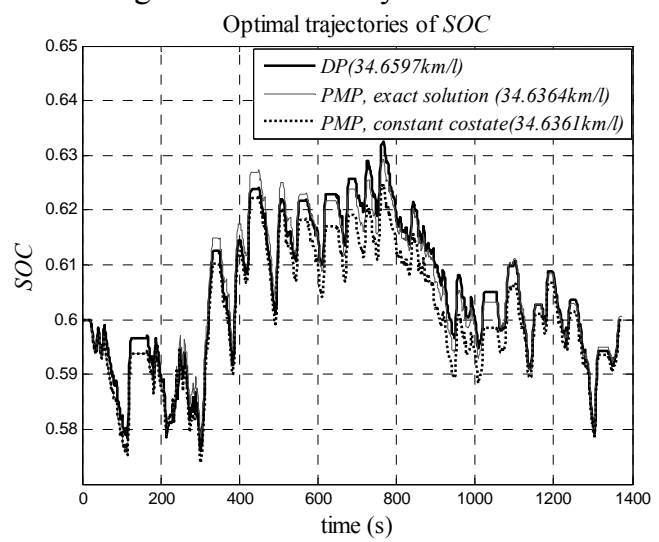

Fig. 11. Optimal results from DP, PMP, and PMP with a constant costate under FUDS.

Mathematically, we would have thought that DP would be superior to PMP and that the exact solution of PMP would be better than the solution of PMP with a constant costate. Based on several other results of our numerical simulations, we can conclude that the fuel economies are not significantly different across the three techniques (see Table 3).

Table 3. Optimal fuel-economy results from the three techniques under various driving schedules. Some results from DP are less than those from PMP because of truncation and numerical errors.

\begin{tabular}{c|c|c|c}
\hline \multirow{2}{*}{ cycle name } & \multirow{2}{*}{ DP $(\mathrm{km} / \mathrm{l})$} & \multicolumn{2}{|c}{ PMP $(\mathrm{km} / \mathrm{l})$} \\
\cline { 3 - 4 } & & Exact solution & Constant $p$ \\
\hline ece_eudc & 30.6576 & 30.6668 & 30.6663 \\
\hline nedc & 32.7784 & 32.7861 & 32.7790 \\
\hline Japan1015 & 37.7861 & 37.7726 & 37.7725 \\
\hline us06 & 21.2126 & 21.0845 & 21.0839 \\
\hline udds truch & 32.3003 & 32.3328 & 32.3317 \\
\hline Manhattan & 29.6651 & 29.6604 & 29.6602 \\
\hline Newyork bus & 24.1240 & 24.1190 & 24.1189 \\
\hline
\end{tabular}

As mentioned above, the results from PMP with constant costates show that the assumptions of the battery are effective in the optimal-control problem of a charge-sustaining HEV. In conclusion, an instantaneous optimal control algorithm using a single constant equivalent parameter can be close to the globally optimal solution when 1) the value of the parameter is properly selected and 2) the battery SOC operates within a proper range, as a result of which the voltage and the resistance do not vary very much.

\section{CONCLUSION}

The main purpose of this paper is to propose a PMP (Pontryagin's minimum principle) algorithm to control hybrid vehicles in optimality. The PMP algorithm can be used for real-time optimal control because it is based on the instantaneous minimization of the Hamiltonian. We proved that the optimal control based on PMP can be a global optimal control under the assumptions of the battery, which are reasonable in a charge-sustaining optimal control problem of HEVs. Based on the assumption, the costate of PMP can be considered as a constant parameter; it simplifies the optimalcontrol problem. In simulation results, the optimal control results from PMP are almost the same as the globally optimal control results. Though we use a constant costate for PMP, the results are still excellent when compared to the global optimal results. In this paper, we just show that PMP is useful as an optimal solution for HEV problems. However, our study is ongoing to find out the correlation between the driving cycle and the costate from a number of optimal results. The essential preconditions to enable the correlation are that 1) the optimal costate can be a simple parameter, not a complex time-varying function and 2) optimal control based on PMP with a constant costate guarantees optimality and especially, global optimality. We demonstrated these two facts, which is the most important contribution of this paper.

\section{ACKNOWLEDGMENT}

This work was supported by the Korea Research Foundation Grant funded by the Korean Government (MOEHRD). (KRF-2007-612-D00081)

\section{REFERENCES}

[1] C. C. Lin, H. Peng, J. W. Grizzle, J. Kang, "Power Management Strategy for a Parallel hybrid Electric Truck," IEEE Trans Control Syst. Technol. Vol. 11, No., 6, Nov. 2003, pp. 839-849.

[2] J. Liu, H. Peng, "Control Optimization for a Power-Split Hybrid Vehicle," in Proc. 2006 American Control Conf., Minneapolis, Minnesota, 2006, pp. 466-471.

[3] Z. Han, Z. Yuan, T. Guangyu, C. Quanshi, C. Yaobin, "Optimal Energy Management Strategy for Hybrid Electric Vehicles," in Proc. SAE paper, 2004-01-0576, 2004.

[4] S.I. Jeon, S.T. Jo, Y.I. Park, and J.M. Lee, "Multi-mode driving control of a parallel hybrid electric vehicle using driving pattern recognition, " ASME J. Dynamic Syst., Meas., Control, vol. 124, pp.141-149, 2002.

[5] C. C. Lin, H. Peng, J. W. Grizzle, "A Stochastic Control Strategy for Hybrid Electric Vehicles," in Proc. 2004 American Control Conf., Boston, MA, 2004, pp. 4710-4715.

[6] S. Delprat, T. M. Guerra, "Control Strategies for Hybrid Vehicles: Optimal Control," in Proc. VTC Fall 2002, IEEE, 2002, pp. 1681-1685. [7] S. Delprat, J. Lauber, T. Marie, J. Rimaux, "Control of a Parallel Hybrid Powertrain: Optimal Control," IEEE Trans. Veh. Technol., Vol. 53, No. 3, May 2004, pp. 872-881.

[8] G. Paganelli, S. Delprat, T. M. Guerra, J. Rimaux, J. J. Santin, "Equivalent Consumption Minimization Strategy for Parallel Hybrid Powertrains," in Proc. VTC Spring 2002, IEEE, 2002, pp. 2076-2081.

[9] C. Musardo, G. Rizzoni, B. Staccia, "A-ECMS: An Adaptive 
Algorithm for Hybrid Electric Vehicle Energy Management," in Proc. 44th IEEE Conf. Decision Control, 2005 European Control Conf., Seville, Spain, 2005, pp. 1816-1823.

[10] L. Guzzella and A. Sciarretta, Vehicle Propulsion Systems. Introduction to Modeling and Optimization. Berlin: Springer-Verlag, 2005. p.208-225

[11] G. Rousseau, D. Sinoquet, P. Rouchon, "Constrained Optimization of Energy Management for a Mild-Hybrid Vehicle," Oil-Gas Science and Technology, IFP, Vol. 62, No. 4, 2007, pp. 623-624.

[12] X. Wei, L. Guzzella, V. I. Utkin, G. Rizzoni, "Model-Based Fuel Optimal Control of Hybrid Electric Vehicle Using of Hybrid Electric Vehicle Using Variable Structure Control Systems," ASME J. Dynamic Syst., Meas., Control, vol. 129, No. 1, January 2007, pp. 13-19.

[13] L. Serrao, G. Rizzoni, "Optimal Control of Power Split for a Hybrid Electric Refuse Vehicle," in Proc. 2008 American Control Conf., Seattle, WA, 2008, pp 4498-4503.

[14] A. Sciarretta, L. Guzzella, "Control of Hybrid Electric Vehicles," IEEE Control. Syst. Mag., April, 2007, pp. 60 70.

[15] Donald E. Kirk, Optimal Control Theory. An Introduction, Prentice Hall, 1970, pp. 209.

[16] H. L. Benford, M. B. Leising, "The Lever Analogy: a New Tool in Transmission Analysis," SAE technical paper, 810102, 1981.

[17] A. Sciarretta, M. Back, L. Guzzella, "Optimal Control of Parallel Hybrid Electric Vehicle," IEEE Trans. Control Syst. Technol., Vol. 12, No. 3, May 2004, pp. 352-363.

[18] V. Jeyakumar, "A Note on Strong Duality in Convex Semidefinite Optimization: Necessary and Sufficient Conditions," Journal of Optimization Letters, Vol. 2, No. 1, January 2008, pp. 15-25.

[19] Donald E. Kirk, Optimal Control Theory. An Introduction, Prentice Hall, 1970, pp. 237.

[20] Donald E. Kirk, Optimal Control Theory. An Introduction, Prentice Hall, 1970, pp. 343.

[21] Vadim F. Krotov, Global Methods in optimal control theory, Marcel Dekker, Inc., 1996, pp. 140.

[22] K. Ahn, S. Cho, S. W. Cha, "Optimal Operation of the Power-split Hybrid Electric Vehicle Powertrain," in Proc. IMechE, Part D: J. Automobile Engineering, Vol. 222, No. 5, 2008, pp. 789-800.

[23] D. P. Bertsekas, Nonlinear Programming: Second Edition, Belmont, Athena Scientific, 1999, pp. 357.

[24] P. Pisu, G. Rizzoni, "A Comparative Study of Supervisory Control Strategies for Hybrid Electric Vehicles, "IEEE Trans. Control Syst. Technol., Vol. 15, No. 3, May 2007, pp. 506-518. 